\title{
Analysis of the Interactions of Botanical Extract Combinations Against the Viability of Prostate Cancer Cell Lines
}

\author{
Lynn S. Adams, Navindra P. Seeram, Mary L. Hardy, Catherine Carpenter and David Heber
}

Center for Human Nutrition, David Geffen School of Medicine, University of California at Los Angeles, Los Angeles, CA 90095, USA

\begin{abstract}
Herbal medicines are often combinations of botanical extracts that are assumed to have additive or synergistic effects. The purpose of this investigation was to compare the effect of individual botanical extracts with combinations of extracts on prostate cell viability. We then modeled the interactions between botanical extracts in combination isobolographically. Scutellaria baicalensis, Rabdosia rubescens, Panax-pseudo ginseng, Dendranthema morifolium, Glycyrrhiza uralensis and Serenoa repens were collected, taxonomically identified and extracts prepared. Effects of the extracts on cell viability were quantitated in prostate cell lines using a luminescent ATP cell viability assay. Combinations of two botanical extracts of the four most active extracts were tested in the 22Rv1 cell line and their interactions assessed using isobolographic analysis. Each extract significantly inhibited the proliferation of prostate cell lines in a time- and dose-dependent manner except $S$. repens. The most active extracts, $S$. baicalensis, D. morifolium, G. uralensis and $R$. rubescens were tested as two-extract combinations. $S$. baicalensis and D. morifolium when combined were additive with a trend toward synergy, whereas $D$. morifolium and $R$. rubescens together were additive. The remaining two-extract combinations showed antagonism. The four extracts together were significantly more effective than the two-by-two combinations and the individual extracts alone. Combining the four herbal extracts significantly enhanced their activity in the cell lines tested compared with extracts alone. The less predictable nature of the two-way combinations suggests a need for careful characterization of the effects of each individual herb based on their intended use.
\end{abstract}

Keywords: botanical - isobologram - PCSPES - prostate cancer

\section{Introduction}

In Chinese medicine, formulas such as Huangquin-tang (1) which contain combinations of natural ingredients are prescribed for the treatment of prostate cancer. These combinations are intended to attain increased potency due to synergistic interactions between the individual components, such that the combination is superior to individual herbal treatment alone. Creating herbal mixtures as opposed to utilizing a single, active herb can present the benefit of lower

For reprints and all correspondence: Lynn S. Adams, University of California at Los Angeles, 900 Veteran Avenue, Los Angeles, CA 90095-1742, USA. Tel: +1-310-825-6938; Fax: +1-310-206-5264; E-mail: ladams@mednet.ucla.edu doses of each individual herb, thus reducing the incidence of dose-related side effects at the same bioeffective dose of a single herb. This treatment philosophy is consistent with the systemic theory of treating chronic diseases, which advocates the inclusion of many active organic substances in formulas to not only treat the disease but to create homeostasis within the body (2).

Interactions between organic substances have been tested in limited fashion in herbal combinations and have demonstrated the possibility of antagonism as well as synergism. In a recent report (3) of eight herbs from the PCSPES formula for prostate cancer, the most cytotoxic herb ( $P$. notoginseng) was varied with respect to the rest of the mixture, and isobolographic analysis carried out on the resulting data. The analysis revealed

(C) The Author (2006). Published by Oxford University Press. All rights reserved.

The online version of this article has been published under an open access model. Users are entitled to use, reproduce, disseminate, or display the open access version of this article for non-commercial purposes provided that: the original authorship is properly and fully attributed; the Journal and Oxford University Press are attributed as the original place of publication with the correct citation details given; if an article is subsequently reproduced or disseminated not in its entirety but only in part or as a derivative work this must be clearly indicated. For commercial re-use, please contact journals.permissions@oxfordjournals.org 
antagonism of $P$. notoginseng against the mixture of the other herbs in the formula, even though the complete PCSPES mixture had been shown to effectively inhibit cancer cell viability in several prior studies $(4,5)$. Therefore, the present analysis was designed to further assess the actions and interactions of plant extracts from the PCSPES formula (Scutellaria baicalensis, Rabdosia rubescens, Panax-pseudo ginseng, Dendranthema morifolium, Glycyrrhiza uralensis and Serenoa repens) separately and in combination, in a prostate cancer cell model.

The plants that were acquired were chosen due to either their inclusion in traditional Chinese formulas for prostate cancer or based on published reports of their effects in prostate cancer cell lines and/or patients. Effects that have been reported for these extracts include: the inhibition of proliferation in prostate, oral, colon and breast cancer cells (6), a decrease in PSA and androgen receptor expression in $\mathrm{LNCaP}$ prostate cancer cells, induction of apoptosis via caspase3 activation (7) and immune stimulating properties (8). Other observed effects show the induction of $G_{1}$ and $G_{2} / M$ cell cycle arrest in prostate cancer, leukemia and hepatic cancer cell lines (9-12) and decreased $\mathrm{COX}-2$ and $\mathrm{Bcl}_{2}$ expression in prostate cancer cell lines (13). S. repens, in particular, is widely known for its ability to alleviate frequency of urination in men with benign prostatic hyperplasia (BPH) (14-16). Studies have also shown that treatment with $S$. repens is associated with prostatic epithelial contraction and decreases in tissue dihydrotestosterone (DHT) levels in men with BPH $(17,18)$.

Three prostate cancer cell lines were studied including an immortalized prostate epithelial cell line derived from a histologically normal adult human prostate and two cell lines of differing invasiveness to assess the effects of the botanicals in a model of the multistep process of carcinogenesis. The interactions among the four most potent extracts in the 22Rv1 prostate carcinoma cell line were then analyzed using isobolographic analysis. This method was first introduced by Loewe (19) in 1928 and has been widely utilized in interaction studies of both drugs and herbs (3,20-22). In contrast to traditional means of statistical analyzing dose-effect data which merely shows an effect but does not distinguish between additivity and synergy, isobolographic analysis depicts this difference graphically thus allowing for a visual assessment of the interaction. We then calculated a combination index (CI) for the data, to mathematically compare the observed response with the expected, calculated response. In addition to the two-way combinations, a mixture of the four most potent extracts was also tested in the viability assay and compared with controls and the two-way mixtures.

\section{Methods}

\section{Plant Materials and Extracts}

Plant materials were collected, authenticated, extracted and standardized as previously reported by our laboratory (23).
Briefly, the aerial parts of the Chinese herbs (S. baicalensis, $R$. rubescens, $P$. ginseng, D. morifolium and G. uralensis) were collected and authenticated in China by Phytomedical Research, Inc. (Beijing, China), and extracted by Botanica Biosciences (Ojai, CA, USA), in $70 \%$ ethanol : water. Commercial standards of baicalin, baicalein, chlorogenic acid and fatty acids were purchased from Sigma (St Louis, MO, USA). Ginsenosides and wogonin were purchased from Indofine (Hillsborough, NJ, USA). Commercial standard of oridonin was purchased from Herbstandard, Inc. (Chesterfield, MO, USA). Dried berries of Saw Palmetto (Florida, USA) were extracted sequentially in cold hexane, acetone and then ethanol at the Phytochemistry Laboratory of the Center for Human Nutrition, UCLA, USA. Solvent was removed in vacuo at low temperature to yield hexane, acetone and ethanol extracts, respectively. The Saw Palmetto extract was analyzed for fatty acid content by gas chromatography (GC). The extracts of the Chinese herbs were analyzed for their respective chemical marker compounds (referenced above) by highperformance liquid chromatography as previously reported (23). For cell culture assays, herb extracts were dissolved in DMSO with the exception of $G$. uralensis, which was dissolved in $70 \%$ ethanol, and sterile filtered with a $0.22 \mu \mathrm{m}$ Millex ${ }^{\circledR}$-GP filter unit (Millipore Corporation, Billerica, MA, USA).

\section{Cell Culture}

All cell lines were acquired from American Type Culture Collection (Rockville, MD, USA). 22Rv1 prostate cancer cells were grown in RPMI 1640 containing 10\% fetal bovine serum (FBS) in the presence of $100 \mathrm{U} \mathrm{ml}^{-1}$ of penicillin and $0.1 \mathrm{~g} \mathrm{l}^{-1}$ of streptomycin. RWPE-1 and RWPE- 2 prostate cells were grown in Defined Keratinocyte Serum Free Medium (DKSFM) containing epidermal growth factor (EGF), insulin and fibroblast growth factor (FGF). Cells were incubated at $37^{\circ} \mathrm{C}$ with $95 \%$ air and $5 \% \mathrm{CO}_{2}$. All cells were maintained below passage 20 and used in experiments during the linear phase of growth.

\section{ATP Assay}

Cells were plated in 96-well plates at a density of 5000 cells per well for 22Rv1 cells and 10000 cells per well for RWPE-1 and RWPE-2 cells and allowed to attach for $24 \mathrm{~h}$. Cells were then treated with either media, vehicle control ( $\leq 0.30 \%$ DMSO,$\leq 0.15 \%$ ethanol individually for individual extract tests or in combination where extract mixtures were used) or the appropriate treatments for 24, 48 and $72 \mathrm{~h}$. Viability was measured utilizing the CellTiter-Glo ${ }^{\circledR}$ Luminescent Cell Viability Assay (Promega, Madison, WI, USA). When added to cells, the assay reagent produces luminescence in the presence of ATP from viable cells. Results were read on the Orion Microplate Luminometer (Bertholds Detection Systems, Pforzheim, Germany). Values obtained were subtracted from blank wells containing media and vehicle (no cells) to control for the effects of vehicles on the luminescence 

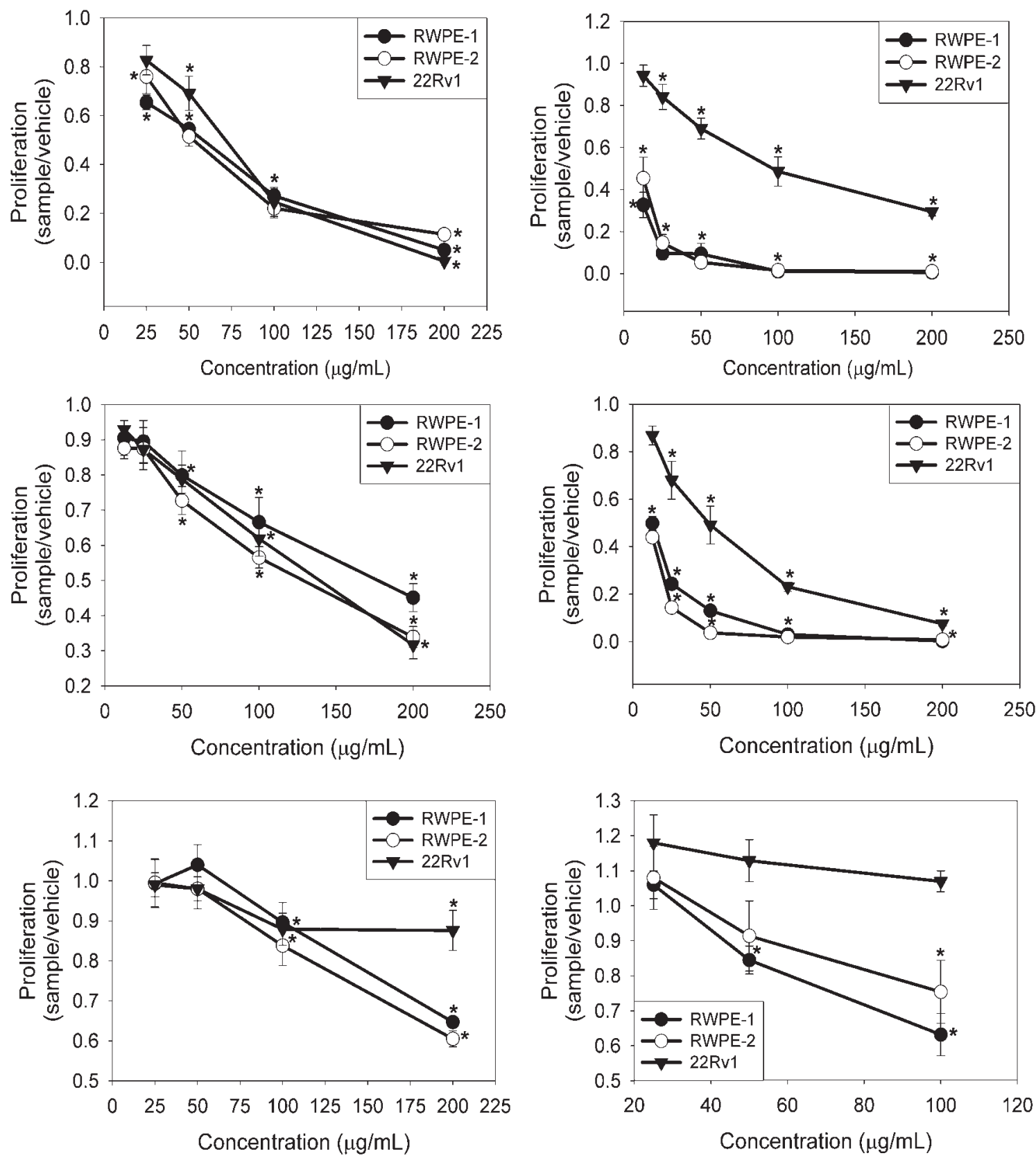

Figure 1. Herbal extracts inhibit proliferation in prostate cell lines. I. S. baicalensis II. G. uralensis III. D. morifolium IV. R. rubescens V. P. ginseng VI. S. repens. Cells were exposed to increasing concentrations of herbal extracts for 48 hours. Cell proliferation was determined via the CellTiter-Glo ${ }^{\circledR}$ Luminescent Cell Viability Assay. Data are expressed as a percentage of vehicle treated controls, mean $\pm \mathrm{SE}\left(\mathrm{n}=3\right.$ ). ${ }^{*} \mathrm{P}<0.01$ compared to vehicle controls.

reagents. Treatment values were then divided by vehicle control values to obtain percent change from control. Due to the large number of data collected from six extracts and three cell lines, only the concentration curve from the $48 \mathrm{~h}$ time point is shown.

\section{Analysis of Antiproliferative Effect and Synergism}

$\mathrm{EC}_{50}$ values for each herbal extract were determined through linear regression with the GraphPad Prism 4 software program (GraphPad Software, Inc., San Diego, CA, USA). In combination experiments, extracts were added at equipotent quantities and at $1 / 2$ and $1 / 4$ fractions of their $\mathrm{IC}_{50}$ value. To assess synergy and antagonism, experimentally derived date was plotted on an isobologram and a CI was determined according to the method of Mertens-Talcott (18).

\section{Statistical Analysis}

Results are presented as mean values \pm SE. Data were analyzed by either one-way ANOVA followed by Dunnet's test $(\alpha=0.05)$ to compare experimental means with controls using GraphPad Prism 4 software. To compare statistical differences between theoretical and experimental means in the 
Table 1. EC50 values of individual herbal extracts in prostate cell lines

\begin{tabular}{|c|c|c|c|}
\hline \multirow[t]{2}{*}{ Extract } & \multicolumn{3}{|c|}{$\mathrm{EC}_{50}$ value $\left(\mu \mathrm{g} \mathrm{ml}^{-1}\right)$} \\
\hline & $22 \mathrm{Rv} 1$ & RWPE-1 & $\overline{\text { RWPE-2 }}$ \\
\hline S. baicalensis & 61.55 & 59.00 & 53.00 \\
\hline G. uralensis & 94.64 & 9.00 & 11.00 \\
\hline D. morifolium & 130.96 & 181.00 & 139.00 \\
\hline R. rubescens & 45.00 & 12.50 & 11.00 \\
\hline$P$. ginseng & $>200$ & $>200$ & $>200$ \\
\hline S. repens & $>200$ & 121.00 & 134.00 \\
\hline
\end{tabular}

isobolograms, Student's $t$-test was performed and $P \leq 0.05$ was accepted.

\section{Results}

\section{Inhibition of Viability with Individual Extracts}

Each of the individual extracts significantly inhibited viability of the three prostate cell lines tested in a dose-dependent manner, with the exception of $S$. repens in the $22 \mathrm{Rv} 1$ cell line $(P \leq 0.001)$ (Fig. 1, I-VI). There was no significant difference between media and vehicle-treated controls as confirmed by two-tailed Student's $t$-test (ethanol: RWPE-1 $P \leq 0.2008$, RWPE-2 $P \leq 0.848,22 \mathrm{Rv} 1 P \leq 0.6098$; DMSO: RWPE-1 $P \leq 0.787$, RWPE-2 $P \leq 0.766,22 \mathrm{Rv} 1 P \leq 0.6098)$. The $\mathrm{EC}_{50}$ values obtained for each extract treatment are shown in Table 1. The four extracts that are most effective in inhibiting cell viability in these three cell lines include $S$. baicalensis, G. uralensis, D. morifolium and $R$. rubescens. Therefore, these extracts were chosen for further analysis as combinations of two herbs and all four together.

\section{Two-way and Four-way Combination Treatments}

Two-way combination studies of the four herbs exhibited varying inhibitory activities in the 22Rv1 cell line (Fig. 2). Inhibition of viability $>50 \%$ of controls was taken to be indicative of additive or enhanced inhibition of growth since all botanical extracts were added at the concentration known to result in 50\% inhibition when tested individually. No significant difference between media and vehicle-treated controls was observed as confirmed by two-tailed Student's $t$-test $(P=0.530)$. The mixture of $D$. morifolium and $S$. baicalensis was the most effective combination treatment, resulting in $72 \%$ inhibition of cell viability compared with vehicle control $(P \leq 0.001)$. Similarly, the combination of D. morifolium and $R$. rubescens inhibited cell viability $57 \%$ compared with vehicle controls $(P \leq 0.001)$. All other two-extract combinations resulted in $<50 \%$ inhibition of cell viability. Nonetheless, all but one of the combinations tested resulted in significant inhibition of cell viability compared with controls. However, when the four herbs were combined into a single treatment (Quad), the result was an $88 \%$ inhibition of viability $(P \leq 0.001)$ compared with vehicle controls (Fig. 2).

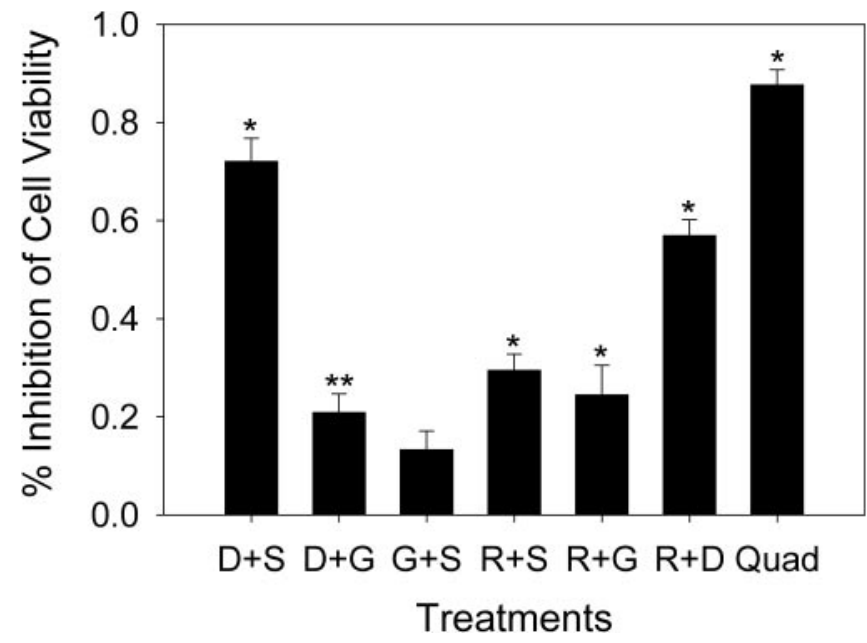

Figure 2. Herbal combinations inhibit proliferation in $22 \mathrm{Rv} 1$ prostate cancer cell line. Cells were exposed to two by two and a four way mixture (Quad) of the four herbs tested at EC50 levels for the 22Rv1 cell line for 48 hours. Data are expressed as a percentage of vehicle treated controls, mean $\pm \operatorname{SE}(n \geq 3)$. Asterisks indicate significance from vehicle controls (single asterisk: $\mathrm{P} \leq$ 0.001, double asterisk: $\mathrm{P} \leq 0.05)$.

\section{Isobolographic Analysis}

Isobolographic analysis was performed utilizing the $\mathrm{EC}_{50}$ values of each botanical extract at a $1: 1$ ratio and at appropriate dilutions of this dose ratio. The dose-effect data and statistics for the isobolograms are shown in Table 2. Plotting the $\mathrm{EC}_{50}$ value of one herb of a combination on the $x$-axis and the other on the $y$-axis yielded the isobolograms shown in Fig. 3. The line connecting the two points on the graph demonstrates all combinations that would theoretically result in additivity. The combination of $D$. morifolium and $R$. rubescens falls on the line of additivity, which indicates an additive reaction with this combination. The S. baicalensis and D. morifolium combination fell below the line of additivity; however, the distance was not significant. The other four combinations all fell above the line of additivity; however, only one was not significant (S. baicalensis and G. uralensis). The remaining three combinations ( $R$. rubescens and G. uralensis, S. baicalensis and $R$. rubescens) were significantly different from the line of additivity $(P<0.05)$ indicating antagonistic interactions.

\section{Combination Index}

Utilizing the $\mathrm{EC}_{50}$ values of each individual herb and the $\mathrm{EC}_{50}$ value of the combinations, the $\mathrm{CI}$ was derived to evaluate the level of interaction between the two herbs (18). Consistent with the results of the isobologram, the combination of S. baicalensis and D. morifolium was somewhat $<1(0.968)$, the combination of $D$. morifolium and $R$. rubescens was slightly $>1$ (1.299) and the CI for the S. baicalensis and G. uralensis combination was 2.034. These are the three combinations that were not significantly different from the theoretical additive dose. Alternatively, S. baicalensis and R. rubescens had a CI of 3.984, G. uralensis and R. rubescens was 7.98, 
Table 2. Dose-effect data and statistics for isobolographic analysis

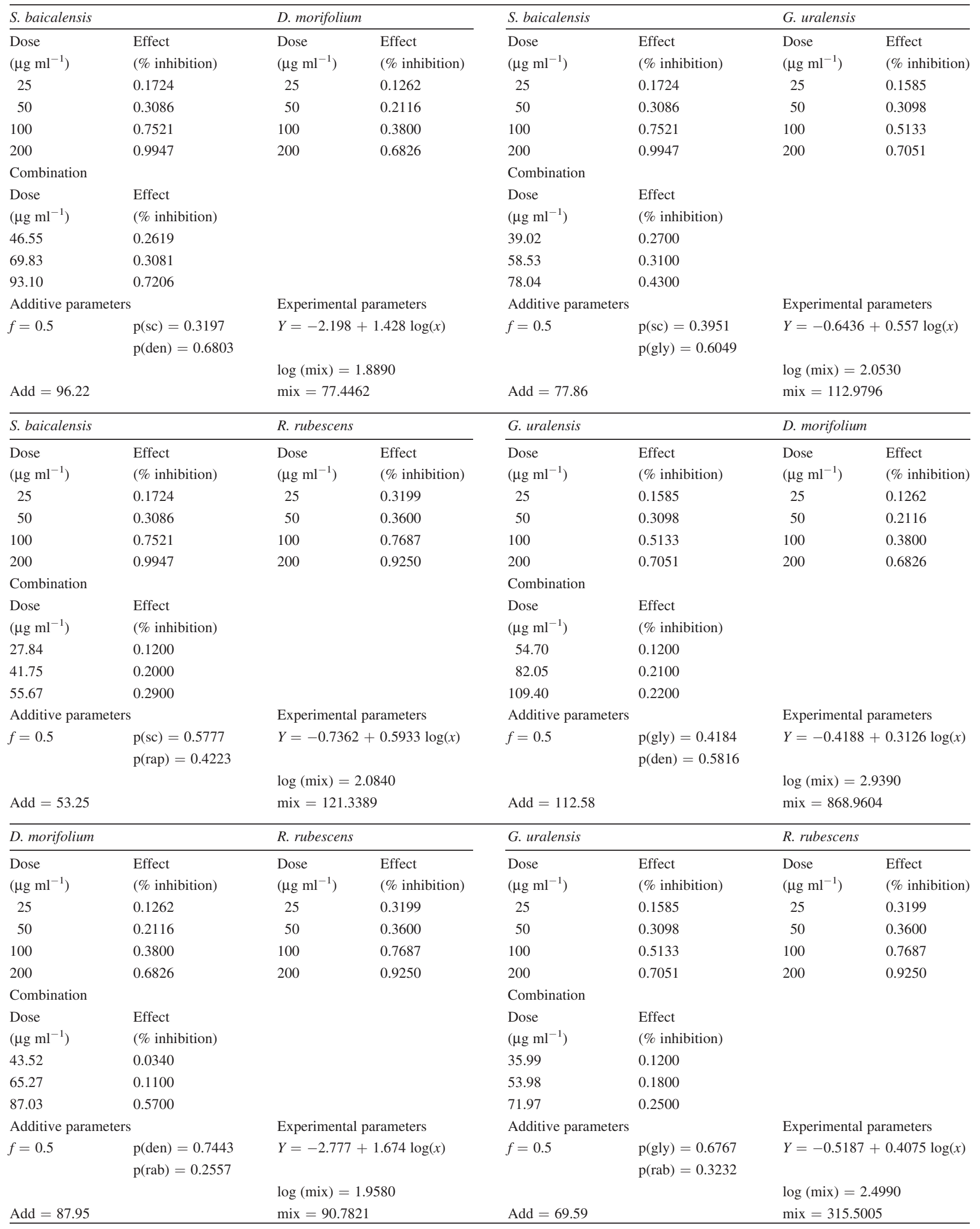



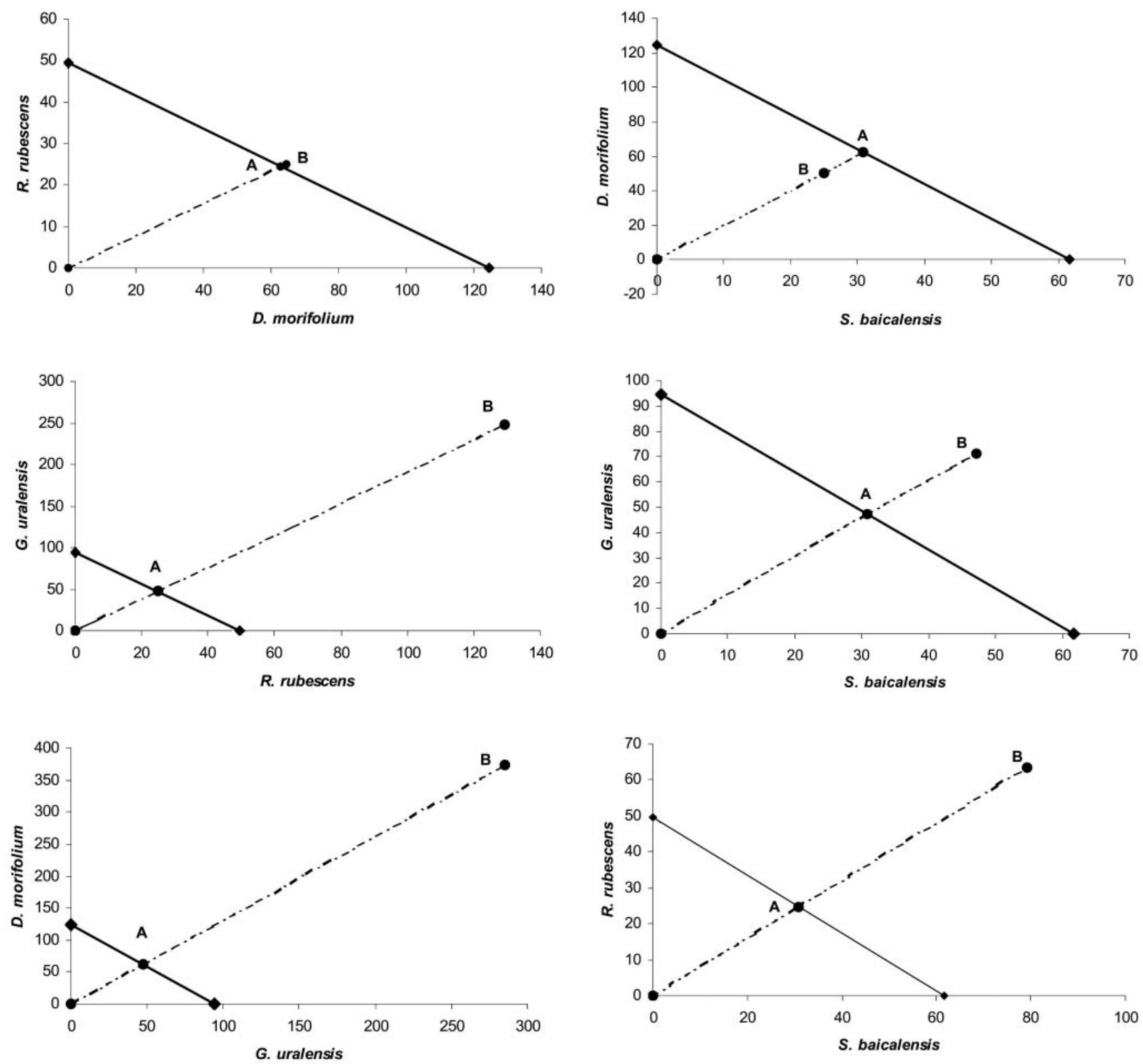

Figure 3. Isobologram demonstrating the interaction of two-by-two herbal combinations. The solid line represents the line of additivity (dose: $\mu \mathrm{g} / \mathrm{mL})$; the broken line represents proportions of the mixtures. Point A represents the calculated additive response where point B indicates the response achieved by testing.

Table 3. Interaction indices for herbal combinations

\begin{tabular}{lcc}
\hline Combination & Combination index & $P$-value \\
\hline S. baicalensis and D. morifolium & 0.968 & 0.571 \\
D. morifolium and R. rubescens & 1.299 & 0.318 \\
S. baicalensis and G. uralensis & 2.034 & 0.242 \\
S. baicalensis and R. rubescens & 3.984 & 0.015 \\
G. uralensis and R. rubescens & 7.980 & 0.031 \\
G. uralensis and D. morifolium & 15.026 & 0.058 \\
\hline
\end{tabular}

and the G. uralensis and D. morifolium combination had a CI of 15.026 (Table 3). These values reflect the isobologram showing that these combinations were significantly greater than the theoretical additive dose, indicating antagonism.

\section{Discussion}

The purpose of this investigation was 2-fold. First, we determined the activity of six widely used natural extracts from a well-known Chinese medicine combination (PCSPES) in different prostate cancer cell lines representing stages in the multistage process of prostate carcinogenesis. Second, we analyzed the interactions between the different herbs in combination, in a selected human prostate epithelial carcinoma cell line (22Rv1). These studies demonstrated varying effects of the four individual herbs on prostate cancer cells in vitro.

$S$. baicalensis, $R$. rubescens, G. uralensis and D. morifolium have been shown to inhibit viability in LNCaP prostate cancer cells in vitro. In this same report, S. baicalensis and 
G. uralensis also decreased PSA and androgen receptor expression (24). Both a flavonoid in S. baicalensis, baicalin, and its aglycone form, baicalein, inhibited viability in DU145, LNCaP and PC-3 prostate cancer cell lines and induced apoptosis via caspase-3 activation (7), and induced $\mathrm{G}_{1}$ and $\mathrm{G}_{2} / \mathrm{M}$ cell cycle arrest in prostate cancer, leukemia and hepatic cancer cell lines (9-12). Oridonin, an extract of $R$. rubescens, decreased $\mathrm{bcl}_{2}$ and increased bax expression in SPCA-1 lung cancer cells (25) and showed antiangiogenic properties in an endothelial cell-based assay for angiogenesis (26).

The results of our initial viability studies confirm the effects observed with these same botanical extracts in previous studies of various prostate cancer cell lines $(4,5)$. We found that botanical extracts from S. baicalensis, G. uralensis, D. morifolium and $R$. rubescens among the six that were tested showed substantial activity in these cell lines. S. repens and $P$. ginseng have been reported to reduce proliferation in $\mathrm{LNCaP}$ prostate cancer cells $(13,27)$. These extracts were the least effective in RWPE-1, RWPE-2 and 22RV1 cells; therefore, the combination studies did not include these extracts. However, cell line specific effects are known to occur and natural compounds may act in either direct or indirect fashion. Indirect action of a compound may affect tissues or compounds outside of the cell and therefore not exhibit detectable activity in a cell culture model. An example of this would be the decrease of tissue DHT levels in vivo after treatment with $S$. repens $(17,18)$.

Isobolographic analysis of the four most potent botanical extracts was studied in the $22 \mathrm{Rv} 1$ cell line which is the most aggressive prostate cancer cell line of the three tested. Our results indicate that some of the two-by-two combinations displayed additive effects suggesting that similar molecular targets or metabolic pathways are involved in their action. The combination of $S$. baicalensis and D. morifolium fell below the line of additivity indicating a trend toward synergy; however, this was not statistically significant.

Four of the combinations displayed clear antagonism. Of the four antagonistic combinations, three contain $G$. uralensis and two contain S. baicalensis. S. baicalensis and G. uralensis together inhibit each other to the point of rendering their action insignificant. It is unlikely that one extract would have an action in the cell that directly opposes inhibition of viability by another extract, as all of them individually result in the inhibition of cell viability. This observation affords us with opportunity for future studies into the mechanisms of these extracts.

More importantly, despite the interactions of the extracts in two-by-two combinations, the overall effect of the absolute mixture was to inhibit cell viability to $88 \%$. This observation is consistent with the idea that combinations of botanical extracts are more effective than isolated components in the mixture. These interactions may depend upon the complimentary nature of different families of compounds in each of the extracts. This principle was demonstrated for food phytochemical extracts in a recent report which demonstrated that tomato powder containing lycopene, phytoene, phytofluene in a complex mixture but not purified lycopene was able to inhibit prostate carcinogenesis in the NMU-testosterone mouse model (28).

The limitations in this work are related to the in vitro method used to evaluate cell viability, which may not reflect the conditions in vivo following absorption and metabolism of the extracts. As previously stated, while direct action may be observed in a cell culture model, indirect actions would unlikely be observed. Nonetheless, these findings provide valuable insight into the effect of the individual botanical extracts on prostate cancer cell viability and encourage investigation into the inhibitory targets and metabolic pathways affected by the botanical materials tested. The results of the isobologram suggest that unknown factors influencing the action of the extracts make their interactions unpredictable. The ratio of one extract to another, the mode of action and the necessity of common cellular resources are only some examples of variables that may determine the efficacy of a mixture. Therefore, comprehension of the activity of each extract when alone and in combination is essential to designing effective mixtures.

\section{Acknowledgements}

Supported by the Center for Dietary Supplement Research: Botanicals (CDSRB) from NIH/NCCAM Grant P50AT00151. L.S.A. is currently on a training grant fellowship from NIH/ NIDDK Award no. 2T32DK007688.

\section{References}

1. Zuo F, Zhou Z, Yan M, Liu M, Xiong Y, Zhang Q, et al. Metabolism of constituents in Huangqin-Tang, a prescription in traditional Chinese medicine, by human intestinal flora. Biol Pharm Bull 2002;25:558-63.

2. Olalde Rangel J, Magarici M, Amendola F, del Castillo O. The systemic theory of living systems. Part IV: systemic medicine-the praxis. Evid Based Complement Alternat Med 2005;2:429-39.

3. Chung V, Tattersall M, Cheung HT. Interactions of an herbal combination that inhibits growth of prostate cancer cells. Cancer Chemother Pharmacol 2004;53:384-90.

4. Kubota T, Hisatake J, Hisatake Y, Said J, Chen S, Holden S, et al. PC-SPES: a unique inhibitor of proliferation of prostate cancer cells in vitro and in vivo. Prostate 2000;42:163-71.

5. Chen S. In vitro mechanism of PC SPES. Urology 2001;58:28-35.

6. Ye F, Xui L, Yi J, Zhang W, Zhang D. Anticancer activity of Scutellaria baicalensis and its potential mechanism. J Altern Complement Med 2002;8:567-72.

7. Chan F, Choi H, Chen Z, Chan P, Huang Y. Induction of apoptosis in prostate cancer cell lines by a flavonoid, baicalin. Cancer Lett 2000;160: 219-28.

8. Haddad P, Azar G, Groom S, Boivin M. Natural health products, modulation of imune function and prevention of chronice diseases. Evid Based Complement Alternat Med 2005;2:513-20.

9. Cheng K, Hou W, Huang Y, Wang L. Baicalin induces differential expression of cytochrome $\mathrm{C}$ oxidase in human lung H441 cell. J Agric Food Chem 2003;51:7276-9.

10. Ikezoe T, Chen S, Heber D, Taguchi H, Koeffler P. Baicalin is a major component of PC-SPES which inhibits the proliferation of human cancer cells via apoptosis and cell cycle arrest. Prostate 2001;49:285-92.

11. Chang W, Chen C, Lu F. Different effects of baicalein, baicalin and wogonin on mitochondrial function, glutathione content and cell cycle progression in human hepatoma cell lines. Planta Med 2002;68:128-32.

12. Hsu S, Hsieh Y, Hsieh W, Chou C. Baicalein induces a dual growth arrest by modulating multiple cell cycle regulatory molecules. Eur J Pharmacol 2001;425:165-71. 
13. Goldmann W, Sharma A, Currier S, Johnston P, Rana A, Sharma C. Saw palmetto berry extract inhibits cell growth and COX-2 expression in prostatic cancer cells. Cell Biol Int 2001;25:1117-24.

14. Gerber GS, Fitzpatrick JM. The role of a lipido-sterolic extract of Serenoa repens in the management of lower urinary tract symptoms associated with benign prostatic hyperplasia. BJU Int 2004;94:338-44.

15. Liu JJ, Huang RW, Lin DJ, Peng J, Wu XY, Pan XL, et al. Antiproliferative effects of oridonin on SPC-A-1 cells and its mechanism of action. J Int Med Res 2004;32:617-25.

16. Capodice J, Bemis D, Buttyan R, Kaplan S, Katz A. Complimentary and alternative medicine for chronic prostatitis/chronic pelvic pain syndrome. Evid Based Complement Alternat Med 2005;2:495-501.

17. Marks LS, Partin AW, Epstein JI, Tyler VE, Simon I, Macairan ML, et al. Effects of a saw palmetto herbal blend in men with symptomatic benign prostatic hyperplasia. J Urol 2000;163:1451-6.

18. Marks LS, Hess DL, Dorey FJ, Luz Macairan M, Cruz Santos PB, Tyler VE. Tissue effects of saw palmetto and finasteride: use of biopsy cores for in situ quantification of prostatic androgens. Urology 2001;57: 999-1005.

19. Loewe S. Die quantitation probleme der pharmakologie. Ergebn Physiol 1928;27:47-187.

20. Pytel YA, Vinarov A, Lopatkin N, Sivkov A, Gorilovsky L, Raynaud JP. Long-term clinical and biological effects of the lipidosterolic extra Serenoa repens in patients with symptomatic benign prostatic hyperplasia. Adv Ther 2002;19:297-306.

21. Tallarida RJ, Stone DJ Jr, Raffa RB. Efficient designs for studying synergistic drug combinations. Life Sci 1997;61:PL:417-25.
22. Mertens-Talcott S, Talcott S, Percival S. Low concentrations of quercetin and ellagic acid synergistically influence proliferation, cytotoxicity and apoptosis in MOLT-4 human leukemia cells. J Nutr 2003;133:2669-74.

23. Sartippour MR, Seeram NP, Heber D, Hardy ML, Norris N, Lu QL, et al. Rabdosia rubescens inhibits breast cancer growth and angiogenesis. Int J Oncol 2005;26:121-7.

24. Luszczki JJ, Borowicz KK, Swiader M, Czuczwar SJ. Interactions between oxcarbazepine and conventional antiepileptic drugs in the maximal electroshock test in mice: an isobolographic analysis. Epilepsia 2003;44:489-99.

25. Liu JJ, Huang RW, Lin DJ, Peng J, Wu XY, Pan XL, et al. Antiproliferative effects of oridonin on SPC-A-1 cells and its mechanism of action. J Int Med Res 2004;32:617-25.

26. Meade-Tollin LC, Wijeratne EM, Cooper D, Guild M, Jon E, Fritz A, et al. Ponicidin and oridonin are responsible for the antiangiogenic activity of Rabdosia rubescens, a constituent of the herbal supplement PC SPES. J Nat Prod 2004;67:2-4.

27. Hsieh T, Wu J. Mechanism of action of herbal supplement PC-SPES: elucidation of effects of individual herbs of PC-SPES on proliferation and prostate specific gene expression in androgen-dependent LNCaP cells. Int J Oncol 2002;20:583-8.

28. Boileau T, Clinton SK, Erdman J. Prostate carcinogenesis in $N$-methyl- $N$-nitrosourea (NMU)-testosterone-treated rats fed tomato powder, lycopene, or energy-restricted diets. J Natl Cancer Inst 2003;95: $1578-86$.

Received August 15, 2005; accepted January 16, 2006 


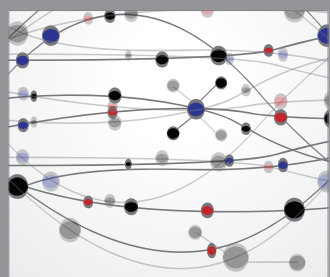

The Scientific World Journal
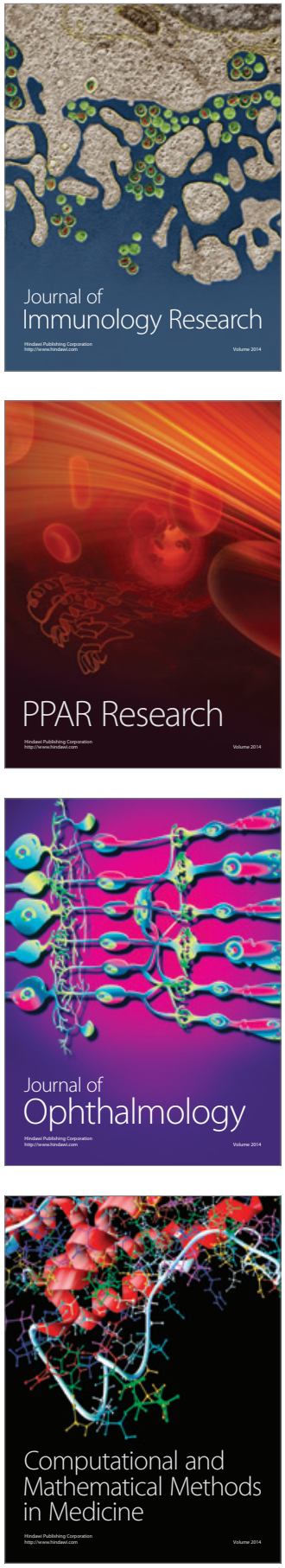

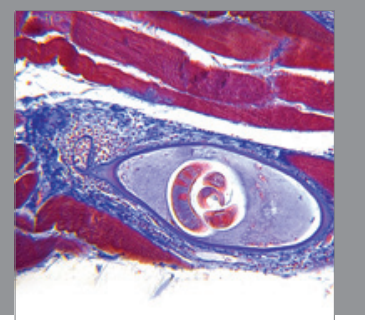

Gastroenterology

Research and Practice
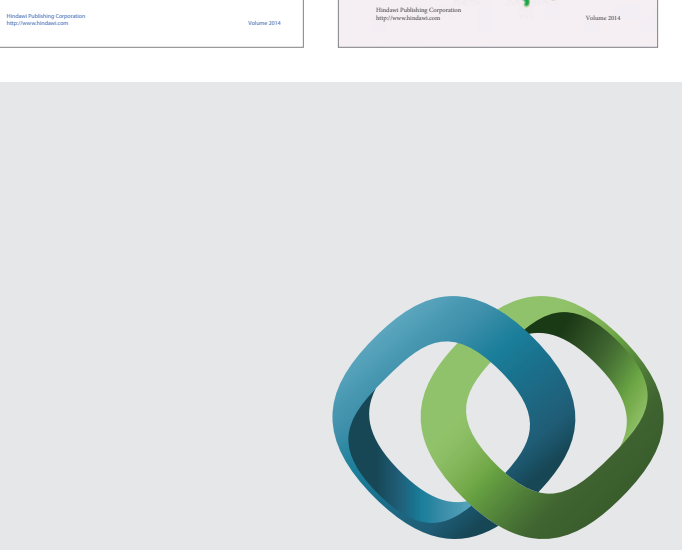

\section{Hindawi}

Submit your manuscripts at

http://www.hindawi.com
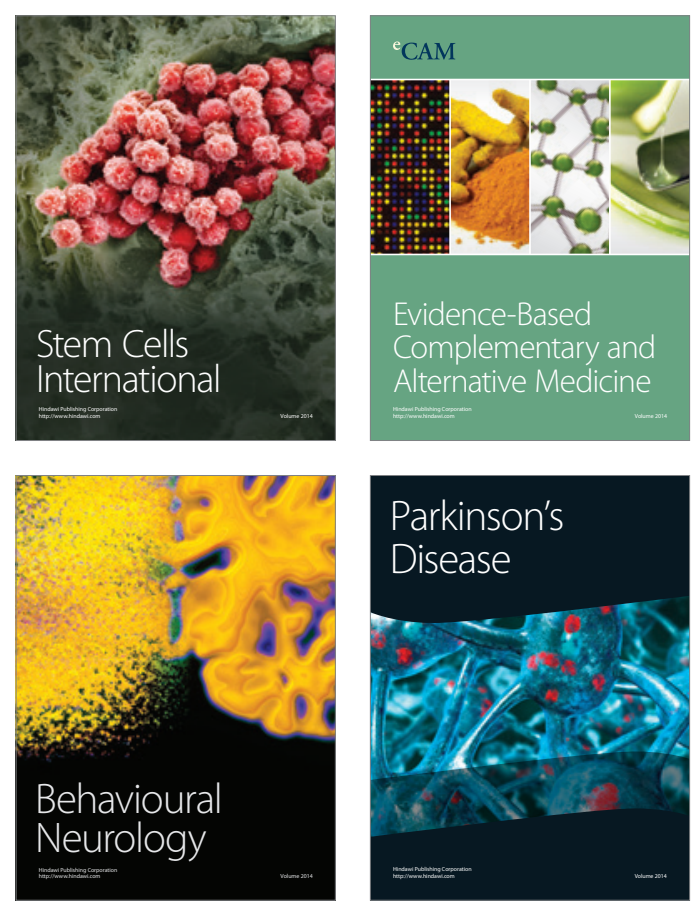

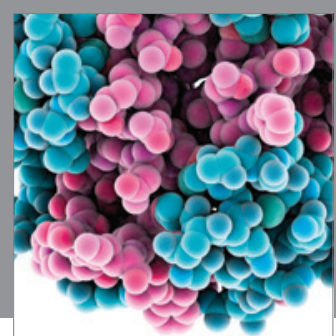

Journal of
Diabetes Research

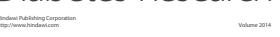

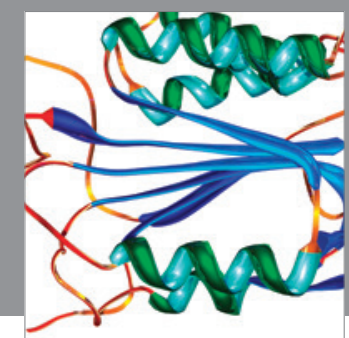

Disease Markers
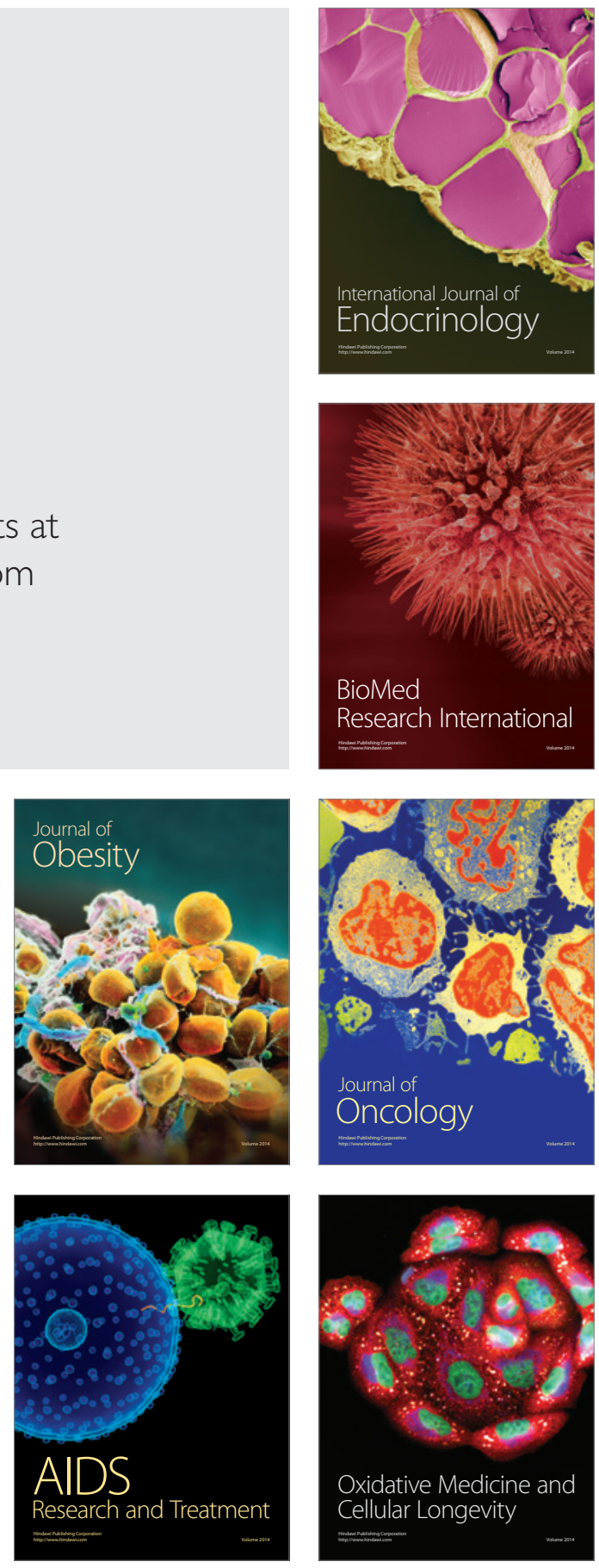